\title{
Cochrane
}

Library

Cochrane Database of Systematic Reviews

\section{Modified dietary fat intake for treatment of gallstone disease} (Protocol)

Madden AM, Trivedi D, Smeeton NC, Culkin A

Madden AM, Trivedi D, Smeeton NC, Culkin A.

Modified dietary fat intake for treatment of gallstone disease (Protocol).

Cochrane Database of Systematic Reviews 2021, Issue 6. Art. No.: CD012608.

DOI: 10.1002/14651858.CD012608.pub2.

www.cochranelibrary.com 
TABLE OF CONTENTS

HEADER 1

ABSTRACT

BACKGROUND

OBJECTIVES

METHODS

ACKNOWLEDGEMENTS

REFERENCES

APPENDICES

WHAT'S NEW

HISTORY

CONTRIBUTIONS OF AUTHORS

DECLARATIONS OF INTEREST

SOURCES OF SUPPORT 
[Intervention Protocol]

\section{Modified dietary fat intake for treatment of gallstone disease}

Angela M Madden 1 , Daksha Trivedi², Nigel C Smeeton ${ }^{2}$, Alison Culkin ${ }^{3}$

1School of Life and Medical Sciences, University of Hertfordshire, Hatfield, UK. ${ }^{2}$ Centre for Research in Primary and Community Care, University of Hertfordshire, Hatfield, UK. ${ }^{3}$ Nutrition \& Dietetic Department, St Mark's Hospital, Harrow, UK

Contact address: Angela M Madden, a.madden@herts.ac.uk.

Editorial group: Cochrane Hepato-Biliary Group.

Publication status and date: Amended to reflect a change in scope (see 'What's new'), published in Issue 6, 2021.

Citation: Madden AM, Trivedi D, Smeeton NC, Culkin A. Modified dietary fat intake for treatment of gallstone disease (Protocol). Cochrane Database of Systematic Reviews 2021, Issue 6. Art. No.: CD012608. DOI: 10.1002/14651858.CD012608.pub2.

Copyright $\odot 2021$ The Cochrane Collaboration. Published by John Wiley \& Sons, Ltd.

\section{A B S T R A C T}

\section{Objectives}

This is a protocol for a Cochrane Review (intervention). The objectives are as follows:

To assess the benefits and harms of modified dietary fat intake in the treatment of gallstone disease. 


\section{B A C K G R O U N D}

Gallstone disease, also known as cholelithiasis, is characterised as hard deposits or stones in the gallbladder and biliary tract. A normally functioning gallbladder stores bile and releases it into the small intestine when it is needed for digestion. Gallstones can develop if the bile contains too much cholesterol or bilirubin, if the gallbladder is dysfunctional, or if the release of bile is impaired. The type of gallstone is defined by its composition and can be divided into two main groups: those that are cholesterol-rich, which are the form predominantly found in people with 'Western' lifestyles; and those that are composed predominantly of bile pigments (Jones 2020).

Recognised risk factors for the disease include female sex, hereditary predisposition, increasing age and body mass index (BMI), rapid weight loss, diabetes, and gastrointestinal and biliary factors, including infection. Prevalence of gallstones varies with the sex and age of the population studied and the diagnostic criteria used; published studies indicate prevalence of between $0.8 \%$ and $64.1 \%$ (Everhart 2002; Ehlin 2003). Prevalence of cholesterol gallstones is generally considered to be increasing as a consequence of nutritional and lifestyle changes, ageing populations, the increasing global prevalence of obesity, and improved diagnostic capabilities (Stinton 2010; Aune 2016).

Gallstones can be diagnosed on the basis of medical history, clinical findings, and imaging. The most appropriate imaging method is abdominal ultrasound imaging, which is supported by high-quality evidence (EASL 2016).

Currently, cholecystectomy, predominantly via a laparoscopic approach, is the standard treatment for symptomatic cholecystolithiasis (Keus 2006; EASL 2016). It is estimated that more than 500,000 people undergo cholecystectomy for symptomatic gallstones each year in the USA (Olsen 1991; NIH 1993; Roslyn 1993).

\section{Description of the condition}

Some people who develop gallstone disease may have no symptoms at all, while others may experience severe abdominal pain (biliary colic), nausea, and vomiting. It is estimated that $2 \%$ to $4 \%$ of people with gallstones develop symptoms each year (Gurusamy 2014). People with gallstone symptoms have a risk of approximately $25 \%$ of developing complications over 10 to 20 years (Tait 1995), which is higher than the complication risk of those who are asymptomatic (Tait 1995; Festi 2010). The risk of complications is also influenced by the location of gallstones, as those lodged in the common bile duct carry a higher risk of complications of approximately $20 \%$ over five years (Tait 1995). Symptoms include cholecystitis, and less commonly, obstructive jaundice, cholangitis, acute pancreatitis, and gangrene of the gallbladder.

\section{Description of the intervention}

Symptomatic gallstone disease is often treated by surgically removing the gallbladder (cholecystectomy), most commonly undertaken laparoscopically (Keus 2006; EASL 2016). While this may be common practice, medical management can also be a firstline treatment. This can include dissolving the gallstones with drug therapy; for example, with ursodeoxycholic acid (Boerlage 2017).

However, traditionally, restricting dietary fat intake was used to reduce the pain associated with gallbladder contractions, rather than dissolving the gallstones. A survey of dietary practice in the UK indicated that people were regularly advised to restrict fat to manage their gallstone disease, but at that time, there was limited empirical evidence to justify this approach (Madden 1992). Mogadam and colleagues also reported that dietary fat restriction was a frequent method of management, but contested the therapeutic relevance of this form of dietary management (Mogadam 1984).

Currently, sources of information for people with gallstone disease advise adherence to low fat, low cholesterol diets, or both (British Liver Trust 2018; Healthline 2018; Patient 2020). This suggests that a dietary intervention is still current treatment for this disease, even though the rationale appears to be uncertain. We consider 'treatment' to mean something that is designed to play a role in the management of the condition rather than to prevent gallstones from forming. A preliminary review of the literature indicates that there is no published evidence of the benefits of a low fat diet compared with standard diet. However, with the increasing prevalence of obesity, there is evidence that people with obesity who are advised to follow weight-reducing diets that incorporate a very low fat diet may be more likely to develop gallstones (Festi 2000), and that diets higher in fat may reduce gallstone risk in adults losing weight (Stokes 2014). We do not anticipate that specific populations would experience different outcomes from interventions.

\section{How the intervention might work}

The rationale for restricting or modifying dietary fat in the treatment of gallstone disease has two putative mechanisms.

First, as dietary fat is a potent stimulator of gallbladder contraction, dietary fat may provoke or exacerbate post-prandial pain. Therefore, hypothetically, restricting dietary fat might reduce pain. However, the gallbladder also contracts spontaneously (Behar 1989), and in response to an intake of mixed meals, protein (Hopman 1985), or cephalic stimulation (Hopman 1987). Furthermore, if restricting dietary fat does lead to a reduction in gallbladder contractions and emptying, it may also increase the risk of gallstone deposition, as lithogenic bile would be retained longer in the gallbladder, thus potentially exacerbating the problem. This mechanism is relevant for gallstones composed of cholesterol and of pigment.

Second, reducing total dietary fat, and particularly saturated fat, leads to a reduction in plasma cholesterol. Lower plasma cholesterol levels may be accompanied by a parallel reduction in biliary cholesterol concentration, which would reduce the precipitation of cholesterol in the bile and decrease the risk of forming cholesterol-rich gallstones (Mendez-Sanchez 2007). This potential mechanism is complicated by the fact that circulating cholesterol levels are more influenced by endogenous cholesterol synthesis than by the intake of dietary cholesterol per se (Lecerf 2011). If this mechanism provides a rationale for the potential treatment of cholesterol-rich stones, it is unlikely to be relevant to the management of stones composed predominantly of pigment.

\section{Why it is important to do this review}

Dietary advice to restrict or modify fat intake, which is currently promoted as treatment for people with gallstones, does not appear to be based on rationalised evidence. While there are general 
health benefits associated with avoiding excessive dietary fat, i.e. reduced risk of obesity and cardiovascular disease, current UK guidelines indicate that specific benefits of a modified diet for the treatment of gallstone disease need clarification (NICE 2014). First, it is important to determine if there are benefits from modified fat intake, or detrimental effects from reduced gallbladder emptying. Second, it would be informative to quantify the amount of fat reduction needed, so that tailored advice could be given, in particular to the minority of people with gallstone disease who are underweight and potentially at risk of malnutrition. We could find no meta-analysis or systematic reviews assessing this topic.

This review will systematically examine the evidence for the dietary management of gallstone disease, clarify the therapeutic benefits and potential risks of dietary interventions as an interim measure while waiting for surgical intervention, and identify the need for future research.

\section{O B J E C T I VES}

To assess the benefits and harms of modified dietary fat intake in the treatment of gallstone disease.

\section{METHODS}

\section{Criteria for considering studies for this review}

\section{Types of studies}

We will include randomised clinical trials assessing benefits and harms of any type of modification of dietary fat intake versus standard care (i.e. no specific additional or alternative intervention), or versus any other type of dietary modification. We will include cluster-randomised clinical trials and cross-over randomised clinical trials, but will only use the data from the first trial period of the latter design to avoid residual effects from the intervention (Higgins 2021a; Higgins 2021b). We will not include quasi-randomised clinical trials, i.e. where a quasi-random method of allocation has been used (alternation, date of birth, or case record number), or observational studies unless they report harms. We will not specifically search for such observational studies for inclusion in this review, which is a known limitation of our review in terms of adverse events. We are aware that the decisions to not search systematically for all observational studies and to extract data on harms might bias our review towards assessment of benefits and might overlook certain harms such as late or rare harms. If we demonstrate benefits from modified dietary fat intake in this systematic review of randomised clinical trials, then a systematic review of the harms of this intervention in observational studies ought to be undertaken (Storebø 2018).

\section{Types of participants}

\section{Inclusion criteria}

We will include participants with gallstone disease (cholesterol, pigment, or mixed) diagnosed using ultrasound, who are receiving a dietary intervention which may or may not have the primary purpose of treating gallstones.

Participants can be male or female, and of any age or ethnic origin.

\section{Exclusion criteria}

We will exclude participants who have been diagnosed with another condition that may compromise dietary fat tolerance, e.g. cholestatic liver disease, short bowel, intestinal failure, or pancreatic insufficiency.

We will exclude groups of participants that include people with and without gallstones if these cannot be analysed separately.

\section{Types of interventions}

\section{Experimental intervention}

The experimental intervention is any type or level of modification of dietary fat intake, providing that it differs from the comparison group.

The experimental intervention might include, for example: restriction of total fat intake, modification of cholesterol intake, long chain fatty acid intake, saturated fat intake, plants sterols and stanols, and fat from specific sources, such as dairy fat or animal fat.

Some trials may have different modes of delivery to the gastrointestinal tract, e.g. oral or enteral nutrition, both of which we will include. However, we will exclude trials where the intervention or comparison is exclusively parenteral, i.e. does not include oral or enteral intake.

We will include trials that test the effects of the frequency and timing of dietary fat intake.

We will also include trials that have three or more dietary interventions, as long as one of the groups contains a form of dietary modification as described above, and we will take account of additional groups during the analysis, as described below (Unit of analysis issues).

We will include trials that include a co-intervention, such as drugs or other dietary (non-fat) components, e.g. psyllium or soluble fibre (Ganji 1994; Theuwissen 2008), providing that the trial groups have received the same proportion of drug or other dietary (non-fat) components in the intervention, or if there are separate groups in the trial in which there have been no drug or other dietary (non-fat) component co-interventions.

\section{Control intervention (comparison group)}

We will compare modified dietary fat intake against standard care (i.e. no specific additional or alternative intervention), or against any other type of dietary modification excluding dietary fat, providing that fat intake can be quantified in both study groups. This could be quantified as either grams of fat per day or per test meal, or expressed as percentage energy. We will analyse studies where the control intervention is standard care (no additional or alternative intervention) separately from those where the control intervention is any other type of dietary modification excluding dietary fat.

\section{Types of outcome measures}

We will collect data with their ranges of follow-up, and will assess all outcomes at the longest follow-up.

\section{Primary outcomes}

- All-cause mortality, at longest follow-up. 
- Proportion of participants with serious adverse events at longest follow-up. Depending on the availability of data, we will attempt to classify adverse events as serious or non-serious. We will record how serious adverse events were assessed in each study according to Good Clinical Practice (GCP). We will define a serious adverse event according to the International Conference on Harmonization (ICH) Guidelines for GCP, as "any untoward medical occurrence that results in death, is life-threatening, requires inpatient hospitalisation or prolongation of existing hospitalisation, results in persistent or significant disability or incapacity, or is a congenital anomaly or birth defect" $(\mathrm{ICH}-$ GCP 1997). This reflects the description used in other Cochrane Review protocols (Markotic 2020). We will specifically report on cholecystitis, pancreatitis, and cholangitis. We will consider any other adverse events to be non-serious (that is, any medical occurrence, not necessarily having a causal relationship with the treatment, but leading to a dose reduction or discontinuation of the treatment).

- Health-related quality of life, assessed using validated tests (e.g. the five-dimension EuroQol (EQ-5D) scale (EuroQol Group 1990), or the 36-item Short Form (SF-36) tool (Garratt 1993), at longest follow-up.

\section{Secondary outcomes}

- Proportion of participants without dissolution or reduction in size of gallstones, at longest follow-up.

- Proportion of participants admitted to hospital for gallstonerelated complications, at longest follow-up.

- Proportion of participants subjected to a surgical intervention, at longest follow-up.

- Proportion of participants with non-serious adverse events, at longest follow-up.

We will not exclude studies that do not report the pre-specified outcomes of the study authors, and if studies do not report important outcomes, we will note this and report it in the Implications for Research part of this review's Conclusions.

\section{Search methods for identification of studies}

\section{Electronic searches}

We will identify trials by searching the Cochrane HepatoBiliary Group Controlled Trials Register (maintained and searched internally by the CHBG Information Specialist via the Cochrane Register of Studies Web), the Cochrane Central Register of Controlled Trials (CENTRAL) in the Cochrane Library, MEDLINE Ovid, Embase Ovid, LILACS (Latin American and Caribbean Health Science Information database) (Bireme), Science Citation Index Expanded (Web of Science), and Conference Proceedings Citation Index - Science (Web of Science; (Royle 2003)). Appendix 1 gives the preliminary search strategies with the expected time spans of the searches. We will include reports of trials in languages other than English, providing we can obtain a reliable translation, which we will do following Cochrane recommendations (Higgins 2021a).

\section{Searching other resources}

Two review authors (AMM and $\mathrm{AC}$ ) will identify other relevant trials by searching reference lists of identified trials and conference proceedings. We will also search online trial registries, such as ClinicalTrial.gov (clinicaltrials.gov/), European Medicines Agency (EMA; www.ema.europa.eu/ema/), World
Health Organization International Clinical Trial Registry Platform (ictrptest.azurewebsites.net/Default.aspx), the Food and Drug Administration (FDA; www.fda.gov), and pharmaceutical company sources, for ongoing or unpublished trials. We will check documents that cite our protocol. We will search for grey literature in the System for Information on Grey Literature in Europe OpenGrey (www.opengrey.eu).

\section{Data collection and analysis}

We will perform the review following Cochrane recommendations (Higgins 2021a). We will use Review Manager Web for the analyses (RevMan web 2020). In case of disagreements between AMM and AC that cannot be resolved by discussion, DT or NS will serve as arbitrator.

\section{Selection of studies}

Two review authors (AMM and AC) will independently review the titles and abstracts of trials identified by the electronic searches and agree on potential publications. We will retrieve the full text of all apparently relevant trials. Two review authors will independently assess the full text of potential trials for inclusion in the review according to the prespecified criteria. We will resolve differences in opinion by discussion. In the event that we cannot resolve differences, we will ask a third author (DT or NS) to provide an opinion. We will keep a record of all included and excluded trials that are selected from the title review.

\section{Data extraction and management}

We will design a data collection form and pilot this on one study. We will then use the adapted form to record study characteristics from the included trials on design, interventions, participants, and outcomes as described in the Criteria for considering studies for this review section above. Two review authors (AMM and AC) will independently extract the data. We will resolve differences in extracted results by discussion, and in the event of no agreement, we will ask a third author (DT or NS) to provide an opinion.

\section{Assessment of risk of bias in included studies}

Two review authors (DT and AMM) will independently assess the risk of bias in each of the included trials. We will resolve any disagreements by consensus or by consulting another review author (NS or $A C$ ) if required. We will assess risk of bias (RoB) using the RoB 2 tool (Sterne 2019; Higgins 2021c), according to the domains defined in the Methodological Expectations of Cochrane Intervention Reviews (MECIR) protocol reporting standard 27 (Higgins 2021d) and methodological studies (Schulz 1995; Moher 2009; Kjaergard 2001; Wood 2008; Savović 2012a; Savović 2012b; Savović 2018). We will use the RoB 2 tool when assessing the effect of assignment to the intervention (Sterne 2019; Higgins 2021c). In order to do this, we will perform analysis based on the intentionto-treat (ITT) principle which includes all randomised participants irrespective of the interventions that participants actually received. Where we can obtain the original data from the investigators, we will perform multiple imputation using the methods described by Jakobsen 2017.

We will use the following five domains to assess bias in the individually randomised trials, including cross-over trials (Higgins 2021b; Higgins 2021c):

- bias arising from the randomisation process; 
- bias due to deviations from intended interventions;

- bias due to missing outcome data;

- bias in measurement of an outcome;

- bias in selection of the reported result.

For trials that allocated clusters of individuals, we will include a sixth domain specific to the trial design to assess bias, i.e. bias arising from the timing of identification and recruitment of individual participants in relation to timing of randomisation (Eldridge 2016).

We will assign one of the three levels of judgement to an overall rating as follows (Naing 2020):

- low risk of bias: the trial is judged to be at low risk of bias for all domains for this result;

- some concerns: the trial is judged to raise some concerns in at least one domain for this result, but is not at high risk of bias for any of the remaining domains;

- high risk of bias: the trial is judged to be at high risk of bias in at least one domain for this result, or the study is judged to have some concerns for multiple domains in a way that substantially lowers confidence in the result.

The overall risk of bias judgement is the same as for the individual domains, such as low risk of bias, some concerns, or high risk of bias. Judging a result to be at a particular level of risk of bias for an individual domain implies that the result has an overall risk of bias at least this severe.

We will use the RoB 2 Microsoft Excel tool to store the data until they can be published in an online repository, which will allow inclusion of the rationale for each judgement for each signalling question for each study for each result (RoB 2 Tool; Sterne 2019).

The overall risk of bias assessments will feed into one domain of the GRADE approach for assessing certainty of a body of evidence (Schünemann 2021).

We will focus on results of the trials that contribute information that users of the review will find most useful. We will, therefore, present the following outcomes in the summary of findings tables:

- all-cause mortality;

- proportion of participants with serious adverse events;

- health-related quality of life.

We will not modify the RoB 2 tool, with the exception of the domains for assessing bias for cluster-randomised clinical trials.

\section{Measures of treatment effect}

We will analyse dichotomous data using risk ratios, converting odds ratios to risk ratios using the standard formula. We will report risk ratios with $95 \%$ confidence intervals $(\mathrm{Cls})$. We will analyse outcomes measured as continuous data, such as patient-reported data that uses a $100 \mathrm{~mm}$ visual analogue scale (Walker 2020), using means and mean differences with their corresponding standard deviations and standard errors, and report these with 95\% Cls. We will consider baseline data and data from all available postintervention time points.

\section{Unit of analysis issues}

We will consider randomised clinical trials that have a parallelgroup design, in which participants are intended to remain in the group to which they were initially assigned. We will consider trials with two or multiple arms eligible.

If trials have non-standard designs or multiple intervention groups, we will consider for each study whether the groups of individuals were randomised together to the same intervention (e.g. clusterrandomised trials; (Higgins 2021b)). We will consider the impact on the analysis of these clustering, matching, or other non-standard design features of the included trials using MECIR Box 6.2.a and MECIR Box 6.2.b (Higgins 2021d).

We will also consider whether individuals underwent more than one intervention (e.g. cross-over trials or simultaneous treatment on each individual), or whether there were multiple observations for the same outcome (e.g. repeated measurements at different time points) (Higgins 2021b).

\section{Dealing with missing data}

We will try to find data on all participants who were randomised, so that we can undertake intention-to-treat analyses, which will include all participants, regardless of adherence or complete follow-up. In cases where outcome data for excluded participants have not been published, we will contact the authors of the trial and request their original data. We will gather information on non-completing participants, including the time and reason for dropping out, as described by the trial authors, and record this on the information extraction form. Where possible, we will incorporate multiple imputation into the analysis (Jakobsen 2017). In addition, we will perform 'worst-best case scenario' and 'bestworst case scenario' analyses for participants lost to follow-up as sensitivity analyses (CHBG Information for authors). A best-worst case analysis is where it is assumed that none of the dropouts lost from the experimental arm, but all of the dropouts lost from the control arm, experienced the outcome. A worst-best case analysis is where it is assumed that all dropouts lost from the experimental arm, but none from the control arm experienced the outcome. Both types of analysis are based on all randomised participants.

\section{Assessment of heterogeneity}

We will assess statistical heterogeneity, which is the presence and extent of between-study variation (Higgins 2021d Box 10.10.a) using the $\mathrm{Chi}^{2}$ test. Where the $\mathrm{P}$ value is less than 0.1 , we will assume there is significant heterogeneity, and quantify heterogeneity using the $I^{2}$ statistic (DerSimonian 1986; Higgins 2002). If intervention trials are combined, errors may arise during the assessment of heterogeneity due to differences in units of analysis (e.g. trials involving cluster randomisation may differ in betweenstudy heterogeneity compared to trials in which individuals are randomised). Although this possibility is largely unexplored, the need to distinguish between the two types of randomised trial has been highlighted (Nyström 2002). To address this, we will use a fixed-effect analysis of comparisons within a trial and then a random-effect analysis between trials.

Methodological heterogeneity, due to differences in how the individual trials are implemented, and clinical heterogeneity, due to differences in participant and intervention characteristics, contribute to the presence and magnitude of statistical 
heterogeneity (Higgins 2003). We will investigate these types of heterogeneity using subgroup analysis.

\section{Assessment of reporting biases}

If at least ten trials are found, we will assess publication bias in terms of treatment effect against trial size by developing a funnel plot using Review Manager Web (RevMan web 2020). We will stratify the funnel plots by risk of bias if we have at least 10 trials for each level of bias (Sterne 2019).

\section{Data synthesis}

\section{Meta-analysis}

We intend to undertake meta-analysis and present the findings according to Cochrane recommendations (Deeks 2021; Higgins 2021a). We will combine data from trials with similar populations, interventions, comparisons, and outcomes. If trials use different scales to measure the same outcome, we will present the standardised mean difference. This measure expresses the size of the intervention effect in each study relative to the variability observed in that study. We will calculate $P$ values for all comparisons where this is possible. We will undertake intentionto-treat analysis wherever possible, so that all randomised participants are included (Jakobsen 2017). Where this is not possible, we will carry out an analysis of available participant cases.

If we include a small number of trials, or if the number of participants is small, we will use the Mantel-Haenszel method for pooling dichotomous data, as this assumes a fixed-effect metaanalysis and is considered an appropriate method (Mantel 1959; Deeks 2021). For continuous data, we will use standardised or mean differences to pool results. If there is no heterogeneity between study findings, we will synthesise and analyse data using a fixedeffect model meta-analysis (DeMets 1987). If this is not possible, we will use the random-effects model (DerSimonian 1986).

If data from included studies preclude meta-analysis, we will undertake one of the following: we will calculate the effect estimate and measure precision from the available statistics if possible (Higgins 2021e); or we will calculate the effect estimate and measure of precision for the same effect measure from the available statistics (Higgins 2021e); or we will transform effect measures (e.g. convert standardised mean differences to an odds ratio; (Deeks 2021)). If none of these are possible, we will tabulate and visually display the results (McKenzie 2021), and will follow the SWiM guidelines for systematic reviews without meta-analysis (Campbell 2020).

\section{Subgroup analysis and investigation of heterogeneity}

Where there are sufficient trials, we will investigate clinical heterogeneity by inspection of the funnel plot(s) (Sterne 2001). We will use a formal statistical test to examine differences among subgroups (Borenstein 2013). This procedure tests for heterogeneity across subgroup results. If there are sufficient data, and irrespective of the presence of any heterogeneity, we plan to perform subgroup analyses. These will include the following.

- Trials at low risk of bias or at low or some concern compared to trials at high risk of bias (because trials at high risk of bias may overestimate beneficial intervention effects or underestimate harmful intervention effects; (Savović 2018)).
- Trials with for-profit support compared to trials without forprofit support (because trials with for-profit support may overestimate beneficial intervention effects or underestimate harmful intervention effects; (Lundh 2018)).

- Trials with participants with acute compared to chronic gallstone disease (because differences in onset of the condition may impact on ability to collect dietary data; (Mou 2019)).

- Trials with participants with high body mass index compared to low body mass index (because body mass index is a measure of obesity and obesity is associated with higher risk of gallstone disease; (Stinton 2010)).

- Trials with participants who are malnourished compared to those who are adequately nourished or over nourished (because people who are malnourished are at higher risk of an inadequate intake associated with a low fat diet; (Madden 2014)).

- Trials with participants with diabetes compared to participants with normoglycaemia (because people with diabetes have a higher risk of gallstone disease; (Aune 2016)).

- Trials with participants with gallstones composed of cholesterol compared to participants with gallstones composed of pigment (because of differences in pathogenesis; (Jones 2020)).

We will undertake analyses separately if trials present data in different units which we cannot convert to a common format (e.g. grams of dietary fat and \% dietary energy from fat).

We will specifically examine the degree of heterogeneity we observe in the results with the $\mathrm{I}^{2}$ statistic, using the guideline that an $I^{2}$ statistic value of $50 \%$ or more indicates a substantial level of heterogeneity (Higgins 2002; Higgins 2003).

We will use subgroup analysis to examine apparent methodological heterogeneity (Deeks 2021; Schünemann 2021).

\section{Sensitivity analysis}

If we identify a sufficient number of randomised trials, we will perform sensitivity analyses to examine the impact of the following factors on effect size:

- excluding trials at high risk of bias;

- size of trials (e.g. large trials);

- trials identified using the following filters: diagnostic criteria; language of publication; source of funding (industry compared to other);

- the impact of participant loss to follow-up by conducting 'worstbest case scenario' and 'best-worse case scenario' analyses.

\section{Trial Sequential Analysis}

We will use Trial Sequential Analysis (TSA) to control the risk of producing random errors due to sparse data and multiple testing of accumulating data (Brok 2008; Wetterslev 2008; Brok 2009; Thorlund 2009; Thorlund 2017; Wetterslev 2017). We will compare our GRADE assessment of imprecision with the TSA assessment of imprecision. We will calculate the required information size (that is, the number of participants needed in a meta-analysis to detect or reject a certain intervention effect) in order to control for random errors (Wetterslev 2008; Wetterslev 2009). For each TSA performed, we will calculate a diversity-adjusted required information size, based on the intervention effect suggested by trials at low risk of bias and an intervention effect of $20 \%$ risk 
reduction, a type I error risk of $2.0 \%$ (the conventional value of $5.0 \%$ divided by 3 , rounded, because of our three primary outcomes), and a type II error risk of $10 \%$ (Wetterslev 2009). We will perform the diversity adjustment using the observed diversity adjustment factor $1 /\left(1-D^{2}\right)$, the heterogeneity estimated by $D^{2}$ among all trials, and with an assumed final diversity of 50\% (Wetterslev 2009). For continuous outcomes we will use a minimal relevant difference equal to SD/2, where SD is the standard deviation of the control group; type I error risk of $2.0 \%$; and a type II error risk of $10 \%$. We will perform meta-analyses sequentially by introducing trials in chronological order (Wetterslev 2017). We will use trial sequential monitoring boundaries to obtain adjusted confidence intervals when the diversity-adjusted required information size and the corresponding number of required trials for the meta-analysis have not been reached (Lan 1983).

If the cumulative Z-value in a particular TSA does not cross the monitoring boundaries for benefit, harm, or futility, we will downgrade our assessment of imprecision in GRADE (see below) by two levels if the accrued number of participants is below $50 \%$ of the diversity-adjusted required information size (DARIS), and by one level if it is between $50 \%$ and $100 \%$ of the DARIS (Jakobsen 2014). We will not downgrade for imprecision if the cumulative Z-value reaches or crosses benefit, harm, futility, or the DARIS (Jakobsen 2014). We will perform TSA with the Trial Sequential Analysis software, version 0.9 .5 .10 beta (Thorlund 2017; TSA 2017).

\section{Summary of findings and assessment of the certainty of the evidence}

We will use the GRADE approach to present data in summary of findings tables as follows. We will present comparisons between the experimental interventions and the control interventions for each primary outcome in a separate summary of findings table.

- All-cause mortality, at longest follow-up.

- Proportion of participants with serious adverse events at longest follow-up.

- Health-related quality of life, assessed using validated tests at a range of follow-ups, including longest follow-up.

We will also provide the maximum follow-up and the range of follow-up for each of the outcomes.

For each outcome, we will include the following information in each summary of findings table.

- The assumed risk; a measure of the typical burden of the outcomes, i.e. the illustrative risk, also called the baseline risk, baseline score, or control group risk.

- The corresponding risk; a measure of the burden of the outcomes after the intervention is applied, i.e. the risk of an outcome in treated/exposed people based on the relative magnitude of an effect and assumed (baseline) risk.

- The relative effect; for dichotomous outcomes, the table will provide the risk ratio, odds ratio, or hazard ratio.

- The number of participants, and the number of trials and their designs.

- Rating of the overall certainty of evidence for each outcome (which may vary by outcome).
- Footnotes or explanations, if needed, to provide explanations about information in the table.

- Comments (if needed).

We will also present the certainty of the evidence for the outcomes reported in the review by considering the within-study risk of bias (methodological quality); indirectness of evidence (population, intervention, control, outcomes); unexplained heterogeneity or inconsistency of results (including problems with subgroup analyses); imprecision of the effect estimate (wide conference intervals; (Jakobsen 2014); and risk of publication bias (Meader 2014; GRADEpro GDT)). Two reviewers (DT and AMM) will work independently to define the evidence as 'high', 'moderate', 'low', or 'very low' certainty. These levels are defined as follows (Schünemann 2013).

- High: we are very confident that the true effect lies close to that of the estimate of the effect.

- Moderate: we are moderately confident in the effect estimate. The true effect is likely to be close to the estimate of the effect, but there is a possibility that it is substantially different.

- Low: our confidence in the effect estimate is limited. The true effect may be substantially different from the estimate of the effect.

- Very low: we have very little confidence in the effect estimate. The true effect is likely to be substantially different from the estimate of effect.

We will resolve disagreement first by discussion and second, if required by, a third reviewer (NS or AC).

\section{ACKNOWLEDGEMENTS}

We thank Dimitrinka Nikolova, Sarah Louise Klingenberg, and Christian Gluud from the Cochrane Hepato-Biliary Group Editorial Team office for their assistance in updating the initial protocol and developing new trial search strategies.

We acknowledge Susan Murray, Royal College of Physicians London, for support in initiating the review and the University of Hertfordshire for the provision of technical support, including computer support and access to literature.

Cochrane Review Group funding acknowledgement: the Danish State is the largest single funder of the Cochrane Hepato-Biliary Group through its investment in the Copenhagen Trial Unit, Centre for Clinical Intervention Research, the Capital Region of Denmark, Rigshospitalet, Copenhagen, Denmark. Disclaimer: the views and opinions expressed in this review are those of the authors and do not necessarily reflect those of the Danish State or the Copenhagen Trial Unit.

Peer Reviewers: Joshua Feinberg, Denmark; Luit Penninga, Denmark; Andrew Anglemyer, New Zealand; Anel Schoonees (search strategies), South Africa

Contact Editor: Ronald L Koretz, USA

Sign-off Editor: Christian Gluud, Denmark

Cochrane Abdomen and Endocrine Network Editor: Rachel Richardson, UK 


\section{REFERE N C E S}

\section{Additional references}

\section{Aune 2016}

Aune D, Vatten LJ. Diabetes mellitus and the risk of gallbladder disease: a systematic review and meta-analysis of prospective studies. Journal of Diabetes and Its Complications 2016;30(2):368-73.

\section{Behar 1989}

Behar J, Lee KY, Thompson WR, Biancani P. Gallbladder contraction in patients with pigment and cholesterol stones. Gastroenterology 1989;97(6):1479-84. [PMID: 2583414]

\section{Boerlage 2017}

Boerlage TC, Haal S, Maurits de Brauw L, Acherman YI, Bruin S, van de Laar AW, et al. Ursodeoxycholic acid for the prevention of symptomatic gallstone disease after bariatric surgery: study protocol for a randomized controlled trial (UPGRADE trial). BMC Gastroenterology 2017;17(1):164. [DOI: 10.1186/ s12876-017-0674-x]

\section{Borenstein 2013}

Borenstein M, Higgins JP. Meta-analysis and subgroups. Prevention Science 2013;14:134-43. [DOI: 10.1007/ s11121-013-0377-7]

\section{British Liver Trust 2018}

British Liver Trust. Gallstones. britishlivertrust.org.uk/wpcontent/uploads/Gallstones-web-version.pdf (accessed 11 November 2020).

\section{Brok 2008}

Brok J, Thorlund K, Gluud C, Wetterslev J. Trial sequential analysis reveals insufficient information size and potentially false positive results in many meta-analyses. Journal of Clinical Epidemiology 2008;61(8):763-9. [PMID: 20042080]

\section{Brok 2009}

Brok J, Thorlund K, Wetterslev J, Gluud C. Apparently conclusive meta-analyses may be inconclusive - trial sequential analysis adjustment of random error risk due to repetitive testing of accumulating data in apparently conclusive neonatal meta-analyses. International Journal of Epidemiology 2009;38(1):287-98

\section{Campbell 2020}

Campbell M, McKenzie JE, Sowden A, Katikireddi SV, Brennan SE, Ellis S, et al. Synthesis without meta-analysis (SWiM) in systematic reviews: reporting guideline. BMJ (Clinical Research Ed.) 2020;368:16890. [DOI: 10.1136/bmj.l6890]

\section{Deeks 2021}

Deeks JJ, Higgins JP, Altman DG, editor(s). Chapter 10: Analysing data and undertaking meta-analyses. In: Higgins JP, Thomas J, Chandler J, Cumpston M, Li T, Page MJ, Welch VA, editor(s). Cochrane Handbook for Systematic Reviews of Interventions version 6.2 (updated February 2021). Cochrane, 2021. Available from www.training.cochrane.org/handbook.

\section{DeMets 1987}

DeMets DL. Methods for combining randomized clinical trials: strengths and limitations. Statistics in Medicine 1987;6(3):341-50

DerSimonian 1986

DerSimonian R, Laird N. Meta-analysis in clinical trials. Controlled Clinical Trials 1986;7(3):177-88.

\section{EASL 2016}

European Association for the Study of Liver Disease (EASL). EASL Clinical Practice Guidelines on the prevention, diagnosis and treatment of gallstones. Journal of Hepatology 2016;65(1):146-81.

\section{Ehlin 2003}

Ehlin AG, Montgomery SM, Ekbom A, Pounder RE, Wakefield AJ. Prevalence of gastrointestinal diseases in two British national birth cohorts. Gut 2003;52(8):1117-21. [DOI: 10.1136/ gut.52.8.1117]

\section{Eldridge 2016}

Eldridge S, Campbell M, Campbell M, Drahota A, Giraudeau B, Higgins J, et al. Revised Cochrane risk of bias tool for randomized trials (RoB 2.0). Additional considerations for cluster-randomized trials. www.riskofbias.info/welcome/ rob-2-0-tool/archive-rob-2-0-cluster-randomized-trials-2016 2016 (accessed 10 November 2020).

\section{EuroQol Group 1990}

EuroQol Group. EuroQol - a new facility for the measurement of health-related quality of life. Health Policy 1990;16(3):199-208. [DOI: 10.1016/0168-8510(90)90421-9]

\section{Everhart 2002}

Everhart JE, Yeh F, Lee ET, Hill MC, Fabsitz H, Howard $\mathrm{BV}$ et al. Prevalence of gallbladder disease in American Indian populations: findings from the Strong Heart Study. Hepatology (Baltimore, Md.) 2002;35(6):1507-12. [DOI: 10.1053/ jhep.2002.33336]

\section{Festi 2000}

Festi D, Colecchia A, Larocca A, Villanova N, Mazzella G, Petroni ML, et al. Review: low caloric intake and gall-bladder motor function. Alimentary Pharmacology \& Therapeutics 2000;14(Suppl 2):51-3. [PMID: 10903004]

\section{Festi 2010}

Festi D, Reggiani ML, Attili AF, Loria P, Pazzi P, Scaioli E, et al. Natural history of gallstone disease: expectant management or active treatment? Results from a population-based cohort study. Journal of Gastroenterology and Hepatology 2010;25(4):719-24. [DOI: 10.1111/j.1440-1746.2009.06146.x]

\section{Ganji 1994}

Ganji V, Kies CV. Psyllium husk fibre supplementation to soybean and coconut oil diets of humans: effect on fat digestibility and faecal fatty acid excretion. European Journal of Clinical Nutrition 1994;48(8):595-7. 


\section{Garratt 1993}

Garratt AM, Ruta DA, Abdalla MI, Buckingham JK, Russell IT. The SF36 health survey questionnaire: an outcome measure suitable for routine use within the NHS? BMJ (Clinical Research Ed.) 1993;306(6890):1440-4. [DOI: 10.1136/bmj.306.6890.1440]

\section{GRADEpro GDT [Computer program]}

McMaster University (developed by Evidence Prime) GRADEpro GDT. Version accessed 4 November 2020. Hamilton (ON): McMaster University (developed by Evidence Prime), 2015. Available at gradepro.org.

\section{Gurusamy 2014}

Gurusamy K, Davidson BR. Gallstones. BMJ (Clinical Research Ed.) 2014;348:g2669. [DOI: 10.1136/bmj.g2669]

\section{Healthline 2018}

Healthline. Gallbladder diet. www.healthline.com/health/ gallbladder-diet (accessed 11 November 2020).

\section{Higgins 2002}

Higgins JP, Thompson SG. Quantifying heterogeneity in a metaanalysis. Statistics in Medicine 2002;21(11):1539-58.

\section{Higgins 2003}

Higgins JP, Thompson SG, Deeks JJ, Altman DG. Measuring inconsistency in meta-analyses. BMJ (Clinical Research Ed.) 2003;327(7414):557-60

\section{Higgins 2021a}

Higgins JP, Thomas J, Chandler J, Cumpston M, Li T, Page MJ, et al, editor(s). Cochrane Handbook for Systematic Reviews of Interventions version 6.2 (updated February 2021). Cochrane, 2021. Available from www.training.cochrane.org/handbook.

\section{Higgins 2021b}

Higgins JP, Eldridge S, Li T, editor(s). Chapter 23: Including variants on randomized trials. In: Higgins JP, Thomas J, Chandler J, Cumpston M, Li T, Page MJ, Welch VA, editor(s). Cochrane Handbook for Systematic Reviews of Interventions version 6.2 (updated February 2021). Cochrane, 2021. Available from www.training.cochrane.org/handbook.

\section{Higgins 2021c}

Higgins JP, Savović J, Page MJ, Elbers RG, Sterne JA. Chapter 8: Assessing risk of bias in a randomized trial. In: Higgins JP, Thomas J, Chandler J, Cumpston M, Li T, Page MJ, Welch VA, editor(s). Cochrane Handbook for Systematic Reviews of Interventions version 6.2 (updated February 2021). Cochrane, 2021. Available from www.training.cochrane.org/handbook.

\section{Higgins 2021d}

Higgins JP, Lasserson T, Chandler J, Tovey D, Thomas J, Flemyng E, et al. Methodological Expectations of Cochrane Intervention Reviews. community.cochrane.org/mecir-manual (accessed 1 February 2021).

\section{Higgins 2021e}

Higgins JP, Li T, Deeks JJ, editor(s). Chapter 6: Choosing effect measures and computing estimates of effect. In: Higgins JP, Thomas J, Chandler J, Cumpston M, Li T, Page MJ, Welch VA, editor(s). Cochrane Handbook for Systematic Reviews of Interventions version 6.2 (updated February 2021). Cochrane, 2021. Available from www.training.cochrane.org/handbook.

\section{Hopman 1985}

Hopman WP, Jansen JB, Lamers CB. Comparative study of the effects of equal amounts of fat, protein, and starch on plasma cholecystokinin in man. Scandinavian Journal of Gastroenterology 1985;20(7):843-7. [PMID: 4048835]

\section{Hopman 1987}

Hopman WP, Jansen JB, Rosenbusch G, Lamers CB. Cephalic stimulation of gallbladder contraction in humans: role of cholecystokinin and the cholinergic system. Digestion 1987;38(4):197-203. [PMID: 3447914]

\section{ICH-GCP 1997}

International Conference on Harmonisation Expert Working Group. International conference on harmonisation of technical requirements for registration of pharmaceuticals for human use. ICH harmonised tripartite guideline. Guideline for good clinical practice CFR \& ICH Guidelines. Vol. 1. Philadelphia (PA): Barnett International/PAREXEL, 1997.

\section{Jakobsen 2014}

Jakobsen J, Wetterslev J, Winkel P, Lange T, Gluud C. Thresholds for statistical and clinical significance in systematic reviews with meta-analytic methods. BMC Medical Research Methodology 2014;14:120.

\section{Jakobsen 2017}

Jakobsen JC, Gluud C, Wetterslev J, Winkel P. When and how should multiple imputation be used for handling missing data in randomized clinical trials - a practical guide with flowcharts. BMC Medical Research Methodology 2017;17(1):162. [DOI: 10.1186/s12874-017-0442-1]

\section{Jones 2020}

Jones MW, Weir CB, Ghassemzadeh S. Gallstones (Cholelithiasis). www.statpearls.com/ArticleLibrary/ viewarticle/22041 (accessed 20 November 2020).

\section{Keus 2006}

Keus F, de Jong JA, Gooszen HG. Laparoscopic versus open cholecystectomy for patients with symptomatic cholecystolithiasis. Cochrane Database of Systematic Reviews 2006, Issue 4. Art. No: CD006231. [DOI: 10.1002/14651858.CD006231]

\section{Kjaergard 2001}

Kjaergard LL, Villumsen J, Gluud C. Reported methodologic quality and discrepancies between large and small randomized trials in meta-analyses. Annals of Internal Medicine 2001;135(11):982-9.

\section{Lan 1983}

Lan KK, DeMets DL. Discrete sequential boundaries for clinical trials. Biometrika 1983;70:659-63. 


\section{Lecerf 2011}

Lecerf JM, Lorgeril M. Dietary cholesterol: from physiology to cardiovascular risk. British Journal of Nutrition 2011;106(1):6-14.

\section{Lundh 2018}

Lundh A, Lexchin J, Mintzes B, Schroll JB, Bero L. Industry sponsorship and research outcome: systematic review with meta-analysis. Intensive Care Medicine 2018;44(10):1603-12.

\section{Madden 1992}

Madden A. The role of low fat diets in the management of gallbladder disease. Journal of Human Nutrition and Dietetics 1992;5:267-73. [DOI: 10.1111/j.1365-277X.1992.tb00165.x]

\section{Madden 2014}

Madden AM, Currie E. Gallbladder disease and nutrition. In: Lomer M, editors(s). Advanced Nutrition and Dietetics in Gastroenterology. 1st edition. Chichester: John Wiley and Son Ltd, 2014:265-72. [DOI: 10.1002/9781118872796]

\section{Mantel 1959}

Mantel N, Haenszel W. Statistical aspects of the analysis of data from retrospective studies of disease. Journal of the National Cancer Institute 1959;22:719-48.

\section{Markotic 2020}

Markotic F, Grgic S, Poropat G, Fox A, Nikolova D, Vukojevic $K$ et al. Antibiotics for adults with acute cholecystitis or acute cholangitis or both. Cochrane Database of Systematic Reviews 2020, Issue 6. Art. No: CD013646. [DOI: 10.1002/14651858.CD013646]

\section{McKenzie 2021}

McKenzie JE, Brennan SE. Chapter 12: Synthesizing and presenting findings using other methods. In: Higgins JP, Thomas J, Chandler J, Cumpston M, Li T, Page MJ, Welch VA, editor(s). Cochrane Handbook for Systematic Reviews of Interventions version 6.2 (updated February 2021). Cochrane, 2021. Available from www.training.cochrane.org/handbook.

\section{Meader 2014}

Meader N, King K, Llewellyn A, Norman G, Brown J, Rodgers M, et al. A checklist designed to aid consistency and reproducibility of GRADE assessments: development and pilot validation. Systematic Reviews 2014;3(82):1-9.

\section{Mendez-Sanchez 2007}

Mendez-Sanchez N, Zamora-Valdes D, Chavez-Tapia NC, Uribe M. Role of diet in cholesterol gallstone formation. Clinica Chimica Acta 2007;376(1-2):1-8. [PMID: 17055469]

\section{Mogadam 1984}

Mogadam M, Albarelli J, Ahmed SW, Grogan EJ, Mascatello VJ. Gallbladder dynamics in response to various meals: is dietary fat restriction necessary in the management of gallstones? American Journal of Gastroenterology 1984;79(10):745-7. [PMID: 6486112]

\section{Moher 2009}

Moher D, Liberati A, Tetzlal J, Altman DG, The PRISMA Group. Preferred reporting items for systematic reviews and meta-analyses: the PRISMA statement. PLoS Medicine 2009;6(7):e1000097. [DOI: 10.1371/journal.pmed.1000097]

\section{Mou 2019}

Mou D, Tesfasilassie T, Hirji S, Ashley SW. Advances in the management of acute cholecystitis. Annals of Gastroenterological Surgery 2019;3(3):247-53. [DOI: 10.1002/ ags3.12240]

\section{Naing 2020}

Naing C, Leong C-O, Aung HH, Mai C-W, Chan EW, Kew ST. Gene therapy for people with hepatocellular carcinoma. Cochrane Database of Systematic Reviews 2020, Issue 9. Art. No: CD013731. [DOI: 10.1002/14651858.CD013731]

\section{NICE 2014}

National Institute of Health and Care Excellence. Gallstone disease: diagnosis and management of cholelithiasis, cholecystitis and choledocholithiasis; 2014. Available at: www.nice.org.uk/guidance/cg188/evidence/fullguideline-193302253.

\section{NIH 1993}

NIH Consensus conference. Gallstones and laparoscopic cholecystectomy. Journal of the American Medical Association 1993;269(8):1018-24. [PMID: 8429583]

\section{Nyström 2002}

Nyström L, Andersson I, Bjurstam N, Frisell J, Nordensjöld B, Rutqvist LE. Long-term effects of mammography screening: updated overview of the Swedish randomised trials. Lancet 2002;359:909-19. [DOI: 10.1016/S0140-6736(02)08020-0]

\section{Olsen 1991}

Olsen DO. Mini-lap cholecystectomy. American Journal of Surgery 1993;165(4):440-3. [PMID: 8480878]

\section{Patient 2020}

Patient. Gallstones diet sheet. patient.info/news-and-features/ gallstones-diet-sheet (accessed 11 November 2020).

\section{RevMan web 2020 [Computer program]}

The Cochrane Collaboration Review Manager W eb (RevMan Web). Version 1.22.0. The Cochrane Collaboration, 2020. Available at revman.cochrane.org.

\section{Roslyn 1993}

Roslyn JJ, Binns GS, Hughes EF, Saunders-Kirkwood K, Zinner MJ, Cates JA. Open cholecystectomy. A contemporary analysis of 42,474 patients. Annals of Surgery 1993;218(2):129-37. [PMID: 8342992]

\section{Royle 2003}

Royle P, Milne R. Literature searching for randomized controlled trials used in Cochrane reviews: rapid versus exhaustive searches. International Journal of Technology Assessment in Health Care 2003;19(4):591-603.

\section{Savović 2012a}

Savović J, Jones HE, Altman DG, Harris RJ, Jüni P, Pildal J, et al. Influence of reported study design characteristics on 
intervention effect estimates from randomised controlled trials: combined analysis of meta-epidemiological studies. Health Technology Assessment 2012;16(35):1-82.

\section{Savović 2012b}

Savović J, Jones HE, Altman DG, Harris RJ, Jüni P, Pildal J, et al. Influence of reported study design characteristics on intervention effect estimates from randomized controlled trials. Annals of Internal Medicine 2012;157(6):429-38.

\section{Savović 2018}

Savović J, Turner RM, Mawdsley D, Jones H, Beynon R, Higgins JP, et al. Association of between risk-of-bias assessments and results of randomized trials in Cochrane Reviews. The ROBES meta-epidemiologic study. American Journal of Epidemiology 2018;187(5):1113-22.

\section{Schulz 1995}

Schulz KF, Chalmers I, Hayes RJ, Altman DG. Empirical evidence of bias. Dimensions of methodological quality associated with estimates of treatment effects in controlled trials. Journal of the American Medical Association 1995;273(5):408-12.

\section{Schünemann 2013}

Schünemann H, Brożek J, Guyatt G, Oxman A, editor(s). GRADE handbook for grading quality of evidence and strength of recommendations. Updated October 2013. The GRADE Working Group; 2013. Available from guidelinedevelopment.org/ handbook.

\section{Schünemann 2021}

Schünemann HJ, Higgins JP, Vist GE, Glasziou P, Akl EA, Skoetz N, et al. Chapter 14: Completing 'Summary of findings' tables and grading the certainty of the evidence. In: Higgins JP, Thomas J, Chandler J, Cumpston M, Li T, Page MJ, Welch VA, editor(s). Cochrane Handbook for Systematic Reviews of Interventions version 6.2 (updated February 2021). Cochrane, 2021. Available from www.training.cochrane.org/handbook.

\section{Sterne 2001}

Sterne JA, Egger M, Smith GD. Systematic reviews in health care: investigating and dealing with publication and other biases in meta-analysis. BMJ (Clinical Research Ed.) 2001;323(7304):101-5. [DOI: 10.1136/bmj.323.7304.101]

\section{Sterne 2019}

Sterne JA, Savović J, Page MJ, Elbers RG, Blencowe NS, Boutron I, et al. RoB 2: a revised tool for assessing risk of bias in randomised trials. BMJ (Clinical Research Ed.) 2019;366:14898. [DOI: 10.1136/bmj.l4898]

\section{Stinton 2010}

Stinton LM, Myers RP, Shaffer EA. Epidemiology of gallstones. Gastroenterology Clinics of North America 2010;39(2):157-69, vii. [PMID: 20478480]

\section{Stokes 2014}

Stokes CS, Gluud LL, Casper M, Lammert F. Ursodeoxycholic acid and diets higher in fat prevent gallbladder stones during weight loss: a meta-analysis of randomized controlled trials. Clinical Gastroenterology and Hepatology 2014;12(7):1090-100.

\section{Storebø 2018}

Storebø OJ, Pedersen N, Ramstad E, Kielsholm ML, Nielsen SS, Krogh HB, et al. Methylphenidate for attention deficit hyperactivity disorder (ADHD) in children and adolescents - assessment of adverse events in non-randomised studies. Cochrane Database of Systematic Reviews 2018, Issue 5. Art. No: CD012069. [DOI: 10.1002/14651858.CD012069.pub2]

\section{Tait 1995}

Tait N, Little JM. The treatment of gall stones. BMJ (Clinical Research Ed.) 1995;311(6997):99-105. [DOI: 10.1136/ bmj.311.6997.99]

\section{Theuwissen 2008}

Theuwissen E, Mensink RP. Water-soluble dietary fibers and cardiovascular disease. Physiology \& Behavior 2008;94(2):285-92.

\section{Thorlund 2009}

Thorlund K, Devereaux PJ, Wetterslev J, Guyatt G, Ioannidis JP, Thabane $L$, et al. Can trial sequential monitoring boundaries reduce spurious inferences from meta-analyses. International Journal of Epidemiology 2009;38(1):276-86.

\section{Thorlund 2017}

Thorlund K, Engstrøm J, Wetterslev J, Brok J, Imberger G, Gluud C. User Manual for Trial Sequential Analysis (TSA); 2017. Available from: ctu.dk/tsa.

\section{TSA 2017 [Computer program]}

Copenhagen Trial Unit TSA - Trial Sequential Analysis. Version 0.9.5.10 Beta. Copenhagen: Copenhagen Trial Unit, 2017. ctu.dk/tsa/downloads/.

\section{Walker 2020}

Walker KF, Chappell LC, Hague WM, Middleton P, Thornton JG. Pharmacological interventions for treating intrahepatic cholestasis of pregnancy. Cochrane Database of Systematic Reviews 2020;7:1-123. [DOI: 10.1002/14651858.CD000493.pub3]

\section{Wetterslev 2008}

Wetterslev J, Thorlund K, Brok J, Gluud C. Trial sequential analysis may establish when firm evidence is reached in cumulative meta-analysis. Journal of Clinical Epidemiology 2008;61:64-75. [PMID: 18083463]

\section{Wetterslev 2009}

Wetterslev J, Thorlund K, Brok J, Gluud C. Estimating required information size by quantifying diversity in random-effects model meta-analyses. BMC Medical Research Methodology 2009;9:86. [PMID: 20042080]

\section{Wetterslev 2017}

Wetterslev J, Jakobsen JC, Gluud C. Trial Sequential Analysis in systematic reviews with meta-analysis. BMC Medical Research Methodology 2017;17(1):39. [PMID: 28264661]

\section{Wood 2008}

Wood L, Egger M, Gluud LL, Schulz KF, Jüni P, Altman DG, et al. Empirical evidence of bias in treatment effect estimates in controlled trials with different interventions and outcomes: 
meta-epidemiological study. BMJ (Clinical Research Ed.) 2008;336:601-5.

\section{References to other published versions of this review \\ Madden 2017}

Madden AM, Trivedi D, Smeeton NC, Culkin A. Modified dietary fat intake for treatment of gallstone disease. Cochrane Database of Systematic Reviews 2017, Issue 3. Art. No: CD012608. [DOI: 10.1002/14651858.CD012608]

\section{AP P E N D I C E S}

\section{Appendix 1. Search strategy}

\begin{tabular}{|c|c|c|}
\hline Database & Time span & Search strategy \\
\hline $\begin{array}{l}\text { The Cochrane Hepa- } \\
\text { to-Biliary Group Con- } \\
\text { trolled Trials Register }\end{array}$ & $\begin{array}{l}\text { Search will be given at } \\
\text { review stage. }\end{array}$ & $\begin{array}{l}\left.\text { (diet* AND fat* AND (restrict* }{ }^{\star} \text { OR modif* or reduc* or low }{ }^{\star} \text { or high }^{\star}\right) \text { ) AND } \\
\text { (cholelithiasis or gallstone* or gall-stone }{ }^{\star} \text { or ((gall or gall bladder or gallblad } \\
\left.\text { der) and stone }{ }^{\star}\right) \text { ) }\end{array}$ \\
\hline
\end{tabular}

Cochrane Central Register of Controlled Trials (CENTRAL) in the Cochrane Library
Latest issue

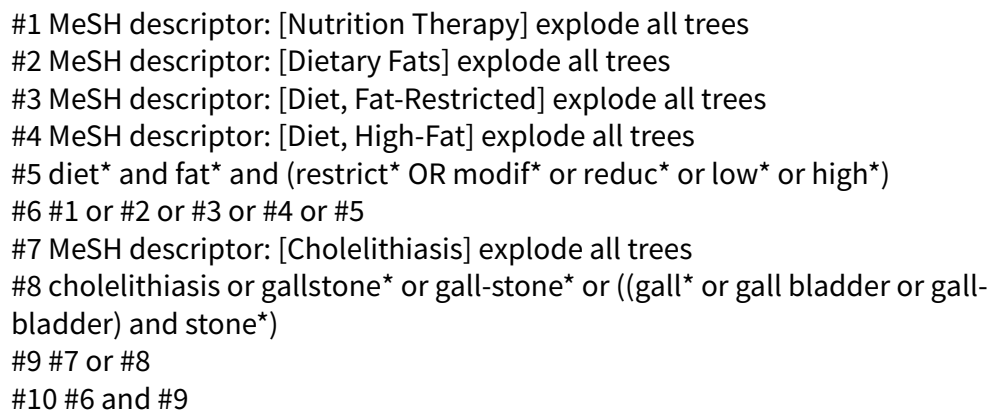

8. (cholelithiasis or gallstone* or gall-stone* or ((gall or gall bladder or gallbladder) and stone $\left.e^{\star}\right) . \mathrm{mp}$. [mp=title, abstract, original title, name of substance word, subject heading word, protocol supplementary concept, rare disease supplementary concept, unique identifier]

\section{7 or 8}

10.6 and 9

11. (randomized controlled trial or controlled clinical trial).pt. or clinical trials as topic.sh. or trial.ti.

12. $\left(\right.$ random $^{\star}$ or blind ${ }^{\star}$ or placebo* or meta-analys $\left.{ }^{\star}\right) \cdot \mathrm{mp}$. [mp=title, abstract, original title, name of substance word, subject heading word, floating subheading word, keyword heading word, organism supplementary concept 
13. 10 and (11 or 12$)$

\begin{tabular}{|c|c|c|}
\hline Embase Ovid & $\begin{array}{l}1974 \text { to the date of the } \\
\text { search }\end{array}$ & $\begin{array}{l}\text { 1. exp diet therapy/ } \\
\text { 2. exp fat intake/ } \\
\text { 3. exp lipid diet/ } \\
\left.\text { 4. (diet* and fat* and (restrict* OR modif* or reduc }{ }^{\star} \text { or low }^{\star} \text { or high }{ }^{\star}\right) \text { ).mp. } \\
\text { [mp=title, abstract, subject headings, heading word, drug trade name, original } \\
\text { title, device manufacturer, drug manufacturer, device trade name, keyword] } \\
\text { 5. } 1 \text { or } 2 \text { or } 3 \text { or } 4 \\
\text { 6. exp cholelithiasis/ } \\
\text { 7. (cholelithiasis or gallstone* or gall-stone* or ((gall or gall bladder or gall- } \\
\left.\text { bladder) and stone }{ }^{\star}\right) \text { ).mp. [mp=title, abstract, subject headings, heading word, } \\
\text { drug trade name, original title, device manufacturer, drug manufacturer, de- } \\
\text { vice trade name, keyword] } \\
\text { 8. } 6 \text { or } 7 \\
\text { 9. } 5 \text { and } 8 \\
\text { 10. Randomized controlled trial/ or Controlled clinical trial/ or trial.ti. } \\
\text { 11. (random }{ }^{\star} \text { or blind }{ }^{\star} \text { or placebo* or meta-analys }{ }^{\star} \text { ).mp. [mp=title, abstract, } \\
\text { heading word, drug trade name, original title, device manufacturer, drug man- } \\
\text { ufacturer, device trade name, keyword, floating subheading word, candidate } \\
\text { term word] } \\
\text { 12. } 9 \text { and ( } 10 \text { or } 11 \text { ) }\end{array}$ \\
\hline $\begin{array}{l}\text { LILACS (Latin American } \\
\text { and Caribbean Health } \\
\text { Science Information } \\
\text { database) (Bireme) }\end{array}$ & $\begin{array}{l}1982 \text { to the date of the } \\
\text { search }\end{array}$ & $\begin{array}{l}\text { (diet\$ and fat\$ and (restrict\$ OR modif\$ or reduc\$ or low\$ or high\$)) [Words] } \\
\text { and (cholelithiasis or gallstone* or gall-stone\$ or ((gall or gall bladder or gall- } \\
\text { bladder) and stone\$)) [Words] }\end{array}$ \\
\hline $\begin{array}{l}\text { Science Citation In- } \\
\text { dex Expanded (Web of } \\
\text { Science) }\end{array}$ & $\begin{array}{l}1900 \text { to the date of the } \\
\text { search }\end{array}$ & 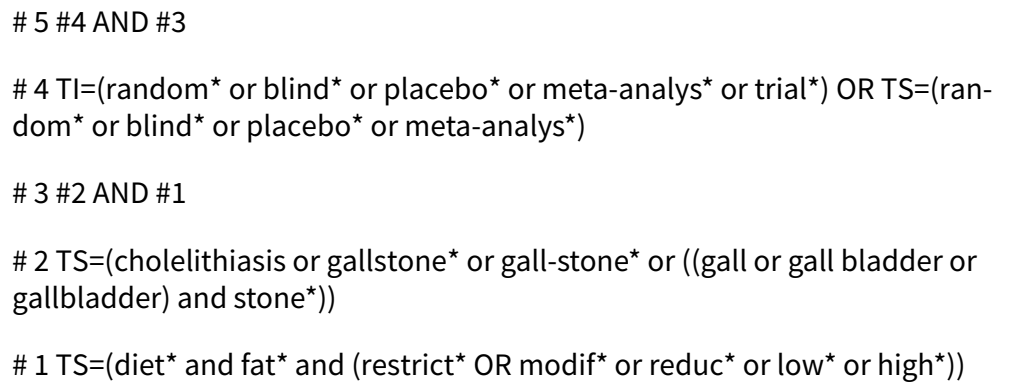 \\
\hline $\begin{array}{l}\text { Conference Pro- } \\
\text { ceedings Citation In- } \\
\text { dex-Science (Web of } \\
\text { Science) }\end{array}$ & $\begin{array}{l}1990 \text { to the date of the } \\
\text { search }\end{array}$ & $\begin{array}{l}\text { \# } 5 \text { \#4 AND \#3 } \\
\text { \# } 4 \text { TI=(random* or blind* or placebo* or meta-analys* or trial*) OR TS=(ran- } \\
\left.\text { dom }^{\star} \text { or blind* or placebo* or meta-analys }\right) \\
\text { \# } 3 \text { \#2 AND \#1 } \\
\text { \# } 2 \text { TS=(cholelithiasis or gallstone* or gall-stone* or ((gall or gall bladder or } \\
\left.\left.\text { gallbladder) and stone }{ }^{\star}\right)\right)\end{array}$ \\
\hline
\end{tabular}


WHAT'S NEW

\begin{tabular}{lll}
\hline Date & Event & Description \\
\hline 3 June 2021 & $\begin{array}{l}\text { New citation required and major } \\
\text { changes }\end{array}$ & $\begin{array}{l}\text { The whole protocol is updated to conform with recent Cochrane } \\
\text { methodology guidelines. }\end{array}$ \\
\hline
\end{tabular}

\section{HIST ORY}

Protocol first published: Issue 3, 2017

\section{CONTRIBUTIONS OF AUTHORS}

All authors contributed to the preparation of this review protocol and approved the final version. AM and AC contributed subject expertise; AM and DT contributed systematic review expertise; NS contributed statistical expertise.

\section{DECLARATIONS OF INTEREST}

AM: none known

DT is an editor with the Cochrane Injuries Group.

NS: none known

AC: none known

\section{SOURCES OF SUPPORT}

\section{Internal sources}

- University of Hertfordshire, UK

Access to electronic resources including peer reviewed journals.

\section{External sources}

- None, UK 